US-96-05

July,1996

hep-th/9609014

\title{
New Algorithm for Tensor Calculation in Field Theories
}

\author{
Shoichi ICHINOSE 巴 \\ Department of Physics, Universuty of Shizuoka, \\ Yada 52-1, Shizuoka 422, Japan
}

\begin{abstract}
Tensor calculation of suffix-contraction is carried out by a C-program. Tensors are represented graphically, and the algorithm makes use of the topology of the graphs. Classical and quantum gravity, in the weak-field perturbative approach, is a special interest. Examples of the leading order calculation of some general invariants such as $R_{\mu \nu \lambda \sigma} R^{\mu \nu \lambda \sigma}$ are given. Application to Weyl anomaly calculation is commented.
\end{abstract}

Keywords: Computer algebra; General relativity; Tensor calculation; Suffix contraction; Graphical representation; Topology of graph

\footnotetext{
${ }^{1}$ E-mail address: ichinose@u-shizuoka-ken.ac.jp
} 


\section{Introduction}

The field theory is described by a Lagrangian which has fields of different spins and their different derivatives: $\varphi, \partial_{\mu} \varphi, \partial_{\mu} \partial_{\nu} \varphi, A_{\mu}, \partial_{\nu} A_{\mu}, h_{\mu \nu}, \partial_{\alpha} h_{\mu \nu}, \cdots(\varphi$ : scalar, $A_{\mu}$ : vector, $h_{\mu \nu}$ : (2nd rank) tensor ). For the n-dimensional flat Euclidean(Lorentzian) space, a general form is a $\mathrm{k}$-th rank tensor ( we call it $k$-tensor in the following, $\mathrm{k}=0,1, \cdots)$ under the global $\mathrm{SO}(\mathrm{n})(\mathrm{SO}(\mathrm{n}-1,1))$ symmetry. In the electro-magnetism, the energy density is given by, in the Euclidean space, an $\mathrm{SO}(\mathrm{n})$-invariant:

$$
\begin{aligned}
\vec{E}^{2}+\vec{B}^{2} & =\frac{1}{2} F_{\mu \nu} F_{\mu \nu} \\
& =\left(\partial_{\mu} A_{\nu}\right)^{2}-\partial_{\mu} A_{\nu} \cdot \partial_{\nu} A_{\mu}, \\
F_{\mu \nu} & =\partial_{\mu} A_{\nu}-\partial_{\nu} A_{\mu} .
\end{aligned}
$$

Similar thing can be said for the Yang-Mills theory. For the curved space, it is valid when we treat a gravitational theory in the weak-field perturbation : $g_{\mu \nu}=$ $\delta_{\mu \nu}+h_{\mu \nu},\left|h_{\mu \nu}\right| \ll 1$. The scalar curvature $R$ is given as

$$
R=\partial_{\mu} \partial_{\mu} h_{\nu \nu}-\partial_{\mu} \partial_{\nu} h_{\mu \nu}+O\left(h^{2}\right)
$$

This is a 0-tensor. Generally physical quantities are expressed by invariants (0tensors) and all suffixes (both derivative-suffixes and field-suffixes) are contracted 2. It is sometimes, however, cumbersome to do calculation involving the suffixcontraction, especially for a higher-spin fields such as gravitational ones.

The importance of high-computational ability in the development of the quantum gravity is historically shown as follows. In 1963 Feynman 1] calculated some scattering amplitudes involving gravitons (,which turned out to be an important finding in gauge theories: Feynman-DeWitt- Faddeev-Popov ghost) by doing algebraic computer calculation. In 1974, 'tHooft and Veltman 2 fully computed the 1-loop ( both matter-field loop and graviton loop) counter-terms of gravitational theories for the first time (by 'finger' calculation using a highly-refined calculational method called 'background-field method'). This result means the quantum gravity is (1-loop) unrenormalizable when matter fields are coupled. It urged theoretical physicists, in the latter half of 70's, to look for or examine other alternative gravitational models such as supergravity. In 1985, Goroff and Sagnotti[3] succeeded in calculating the full 2-loop counter-terms of the pure gravity using a C-language program. Their result was re-confirmed by van de Ven 14 in 1992 by use of a nice algebraic software called 'FORM'[5]. The gauge dependence of their results was examined by [6] using a REDUCE program. The result that 2-loop counter-terms do not vanish on-shell let many people have an opinion that Einstein gravity could not be regarded as the fundamental quantum theory. The present high activity in the string theory is based on these past experiences. The above history of these three and half decays clearly shows, for important development of gravity, good algebraic computer programs and efficient computational techniques play important roles.

\footnotetext{
${ }^{2}$ Generally the summation over all space-suffixes which makes an inner product, such as $A_{\mu} A_{\mu} \equiv$ $\sum_{\mu=1}^{n} A_{\mu} A_{\mu}$, is called Einstein's contraction.
} 
This paper is partly motivated by the analysis of the Weyl anomaly. Compared with the chiral anomaly, the general structure of the Weyl anomaly is not so simple. ( For the chiral one, there is a result for the general n dimension [7].) There exist some analysis [8, 9, 10, 11] and the most popular belief is that they are given by, at each (even) dimension, the Euler term, some Weyl invariants and some 'trivial terms'. The explicit results are obtained only up to the 4 dimensional case [12]. Clearly a way to improve the situation is to explicitly obtain the Weyl anomaly in higher dimensions. We tried it at the $6 \mathrm{dim}$, but we soon realize its calculation involves so much tensor contraction that is difficult in the hand-calculation. Furthermore, the popular algebraic softwares are inconvenient or inefficient for the problem 1 .

In this paper, we present a new algorithm for the tensor contraction and present a C-program ( we call it "WEAKGRAV") using it. Important tensors often have high permutation symmetries with respect to their suffixes. For example, the Riemann tensor has

$$
R_{\mu \nu \alpha \beta}=R_{\alpha \beta \mu \nu}=-R_{\nu \mu \alpha \beta}=-R_{\mu \nu \beta \alpha} .
$$

When we treat 'products' of these tensors, it is most efficient to express the tensors by graphs which have their permutation symmetries 13. All tensor products are completely specified by the topology of graphs. It makes one free from bothering about suffixes. We have only to deal with topology of graphs, not their suffixes. The present algorithm makes use of this idea. In [13], the general tensors in the curved space are treated. In the present paper, we treat global $\mathrm{SO}(\mathrm{n})$ tensors in the flat space.

Generally the local properties of the quantum gravity can be analysed by the perturbation around the flat space. For example, the Weyl anomaly, the effective action and the counterterms are obtained. (We use, in the final form, the fact that they must be invariant under the general-coordinate transformation. This procedure allows us to take into account all orders of the weak-field expansion.) In the case, there appear many terms of "products" of global $\mathrm{SO}(\mathrm{n})$ tensors. The present algorithm is useful in such cases.

The main purpose of this paper is to explain how the above idea about tensorcontraction is realized in the explicit computer calculation. We give two sample calculations. Emphasis is not on the calculated results themselves but on how the results are obtained. Such presentation is good for readers to apply the present algorithm to their own problems.

Finally we describe the main features of the program "WEAKGRAV".

- Computers: all computers running C-program

- Program language used: C-language

- No. of lines including sample data: 300

- Input files needed: indata.dat

\footnotetext{
${ }^{3}$ Most softwares so far treat suffixes directly and the permutation symmetry w.r.t. suffixes is coded by explicitly writing (or declaring) the equality between quantities with the permuted suffixes. This algorithm is not appropriate in the higher dimensions or in higher orders. See Sec.6.
} 


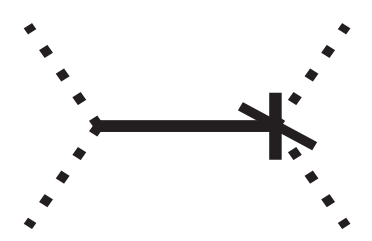

Figure 1: 4-tensor $\partial_{\mu} \partial_{\nu} h_{\alpha \beta}$

- Purpose of program: In the perturbative treatment of gravitational theories, we face many terms with complicated suffix contraction. This program helps to do such calculation very fast. Each term is graphically represented. Topological indices of graphs are utilized in this algorithm. It can be applied to general field theories.

- Restrictions on the complexity of the problem: The program is at a basic level. The contraction terms are restricted to the quadratic type ones: $\partial_{\mu} \partial_{\nu} h_{\lambda \sigma}$. $\partial_{\alpha} \partial_{\beta} h_{\gamma \delta}$. This is, however, sufficient to do Weyl anomaly calculation of $4 \mathrm{dim}$ quantum gravity.

The program "WEAKGRAV" is available on the request to the author's home institute. ( It is restricted only to non-commercial use.)

The content is organized in the following way. In Sec.2 we explain the graphical representation of global $\mathrm{SO}(\mathrm{n})$ tensors, which is used in the explanation of the algorithm. The codes for the tensors are explained in Sec.3. In Sec.4, new quantities called "indices" are introduced and explained. They express the topology of global $\mathrm{SO}(\mathrm{n})$ invariants. We demonstrate the calculation using two simple examples in Sec.5. We conclude in Sec.6. In App.A, all $(\partial \partial h)^{2}$-invariants are graphically shown. Finally, in App.B, we add the input and output data for the sample calculations of Sec.5.

\section{Graphical Representation of Global SO(n) Ten- sors}

In the programming explanation, we use a graphical representation for a tensor because it can clearly express the connectivity of suffixes in a tensor [13, 14]. In this section we explain the minimum amount of the representation necessary for the present programming.

We can graphically express a basic element of the present program: 4-tensor $\partial_{\mu} \partial_{\nu} h_{\alpha \beta}$ as in Fig.1. We call the dotted lines suffix-lines, the rigid line bond and the intersections of two dotted lines and one bond vertices. There are two kinds of vertices: the vertex with a crossing symbol ' $x$ ' and that one without it. The suffixes of derivatives ( $\mu, \nu$ in Fig.1) are associated with those suffix-lines which extend from a vertex without the crossing symbol, whereas the suffixes of fields ( $\alpha, \beta$ in Fig.1) are associated with those which extend from a vertex with it. This graph respects 


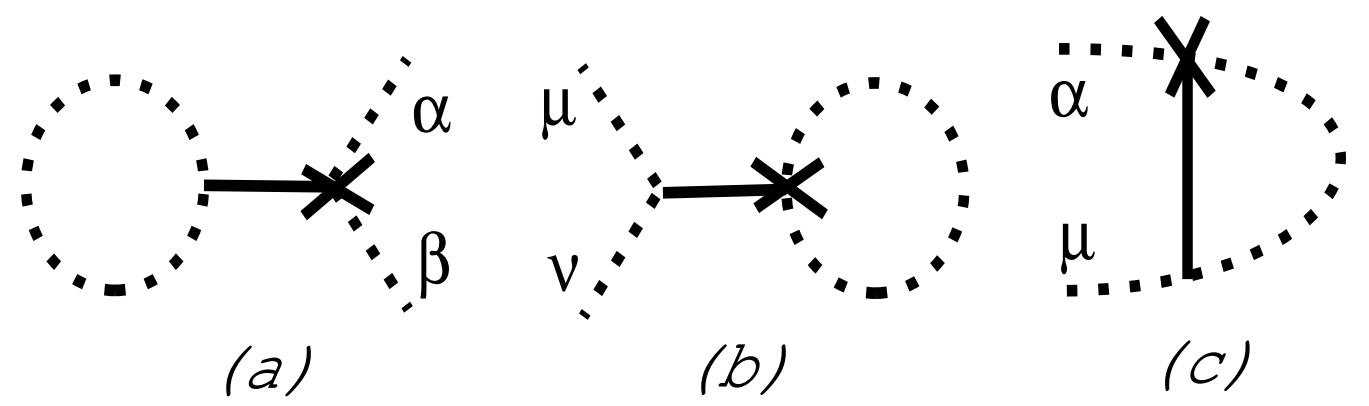

Figure 2: 2-tensors of (a) $\partial^{2} h_{\alpha \beta}$, (b) $\partial_{\mu} \partial_{\nu} h_{\alpha \alpha}$ and (c) $\partial_{\mu} \partial_{\beta} h_{\alpha \beta}$

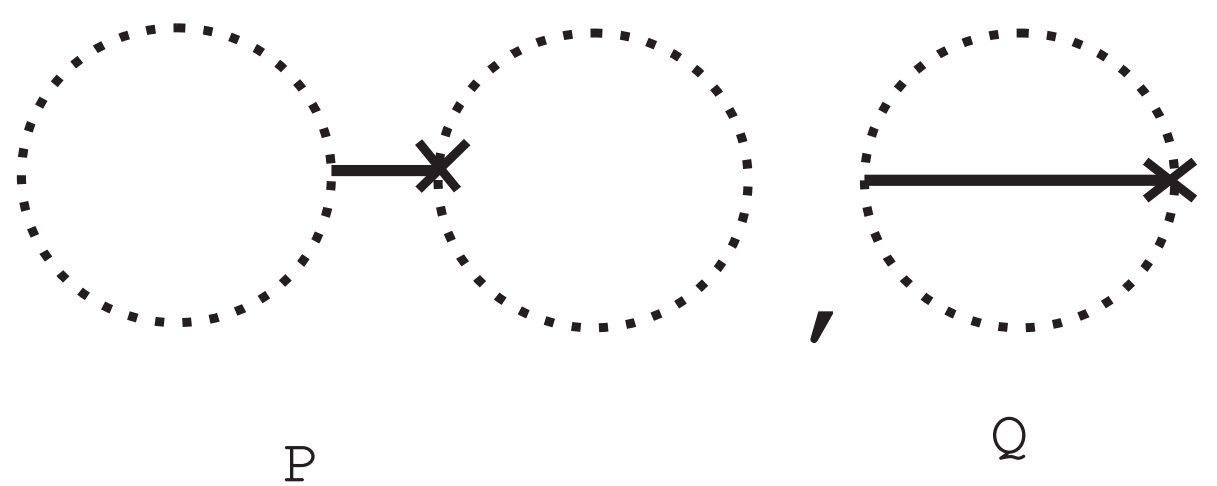

Figure 3: Invariants of $P \equiv \partial_{\mu} \partial_{\mu} h_{\alpha \alpha}$ and $Q \equiv \partial_{\alpha} \partial_{\beta} h_{\alpha \beta}$.

all symmetries of $\partial_{\mu} \partial_{\nu} h_{\alpha \beta}$ w.r.t. the permutation of suffixes: $\partial_{\mu} \partial_{\nu} h_{\alpha \beta}=\partial_{\nu} \partial_{\mu} h_{\alpha \beta}=$ $\partial_{\mu} \partial_{\nu} h_{\beta \alpha}$. The suffix contraction is expressed by connecting the same suffix-lines. For example, 2-tensors : $\partial^{2} h_{\alpha \beta}, \partial_{\mu} \partial_{\nu} h_{\alpha \alpha}, \partial_{\mu} \partial_{\beta} h_{\alpha \beta}$, which are made from Fig.1 by connecting two suffix-lines, are expressed as in Fig.2.

Two independent invariants (0-tensors) $: P \equiv \partial_{\mu} \partial_{\mu} h_{\alpha \alpha}, Q \equiv \partial_{\alpha} \partial_{\beta} h_{\alpha \beta}$, which are made from Fig.2 by connecting the remaining two suffix-lines, are expressed as in Fig.3. They are all possible invariants of $\partial \partial h$-type. Generally all suffix-lines of invariants are closed. Those closed suffix-lines are called suffix-loops.

As for those tensors or invariants which are made from two or more $\partial \partial h$-tensors, their graphical expressions are obtained in the same way. (See the following sections.) We see a tensor and the corresponding graph are one-to-one.

\section{Code for Graphs}

\subsection{Code for $\partial \partial h$-Tensors}

The basic element is the 4-tensor : $\partial_{1} \partial_{2} h_{34}$. Here and in the following we change the Greek symbols, $\mu, \nu, \alpha, \beta$, etc., to the non-negative integer numbers, $0,1,2,3,4$,etc., for the convenience of programming. The four suffixes, characterizing this tensor, are stored in a 3 dimensional array of size $10 \times 2 \times 2: \mathrm{d} 2 \mathrm{~h}[\mathrm{i}][\mathrm{j}][\mathrm{k}]$ as

$\mathrm{d} 2 \mathrm{~h}[\mathrm{i}][0][0]=1$

$\mathrm{d} 2 \mathrm{~h}[\mathrm{i}][0][1]=2$ 


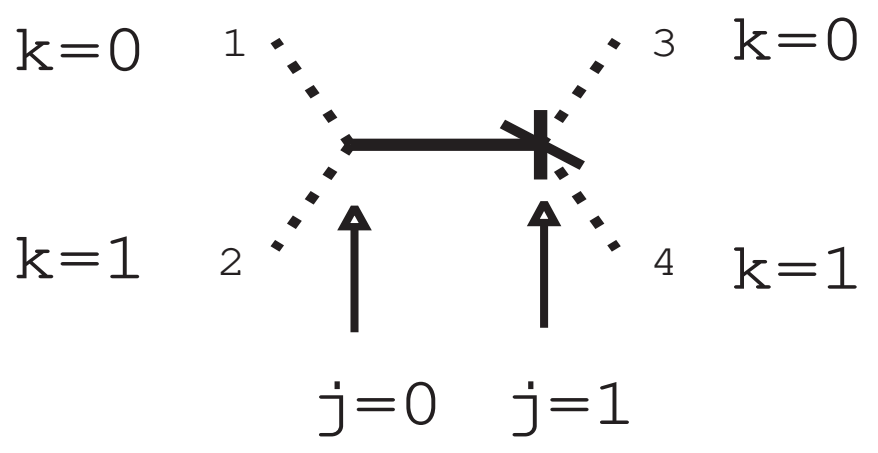

Figure 4: 4-tensor of $\partial_{1} \partial_{2} h_{34}$.

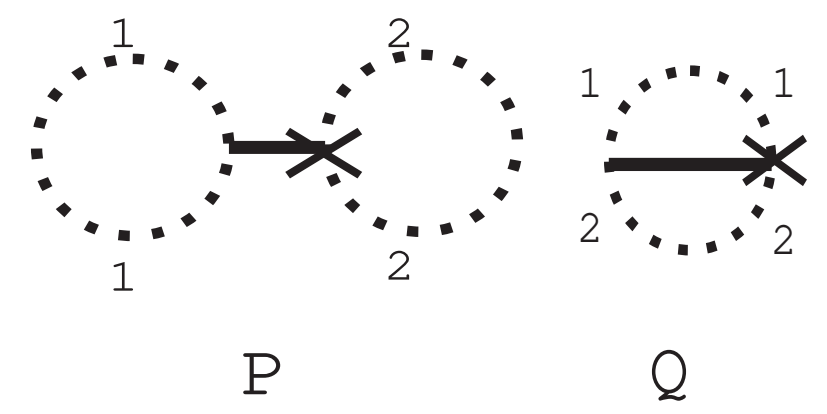

Figure 5: Invariants of $P=\partial_{1} \partial_{1} h_{22}$ and $Q=\partial_{1} \partial_{2} h_{12}$.

$\mathrm{d} 2 \mathrm{~h}[\mathrm{i}][1][0]=3$

$\mathrm{d} 2 \mathrm{~h}[\mathrm{i}][1][1]=4$

where we regard this tensor as "i"-th 'bond' ( $\partial \partial h$-tensor), "j" specifies a chosen vertex-type $(j=0$ for the vertex without $\times, j=1$ for the one with $\times$ ) of the $i$-th $\partial \partial h$-tensor and "k" specifies one of two suffix-lines at each vertex $(i, j)$. f 1 See Fig.4. We call "i","j" and "k" as bond number, vertex-type number and suffix number respectively.

In the examples of Fig.5, we store two invariants, $P=\partial_{1} \partial_{1} h_{22}$ and $Q=\partial_{1} \partial_{2} h_{12}$ in $\mathrm{i}=0$ and $\mathrm{i}=1$ component of $\mathrm{d} 2 \mathrm{~h}[\mathrm{i}][\mathrm{]}[\mathrm{]}$ respectively.
$\mathrm{d} 2 \mathrm{~h}[0][0][0]=1$
$\mathrm{d} 2 \mathrm{~h}[1][0][0]=1$
$\mathrm{d} 2 \mathrm{~h}[0][0][1]=1$$$
\mathrm{d} 2 \mathrm{~h}[1][0][1]=2
$$

\footnotetext{
${ }^{4}$ There exists arbitrariness in the choice of the input data due to the suffix-permutation symmetry of the tensor $\partial_{\mu} \partial_{\nu} h_{\alpha \beta}$. Because the graph identification is programmed in terms of its topology, we may take any one, say,

$\mathrm{d} 2 \mathrm{~h}[\mathrm{i}][0][0]=2$

$\mathrm{d} 2 \mathrm{~h}[\mathrm{i}][0][1]=1$

$\mathrm{d} 2 \mathrm{~h}[\mathrm{i}][1][0]=4$

$\mathrm{d} 2 \mathrm{~h}[\mathrm{i}][1][1]=3$.

${ }^{5}$ As the size of "i", we here take 10 . This allows us to treat up to 10 different $\partial \partial h$-tensors at once.
} 


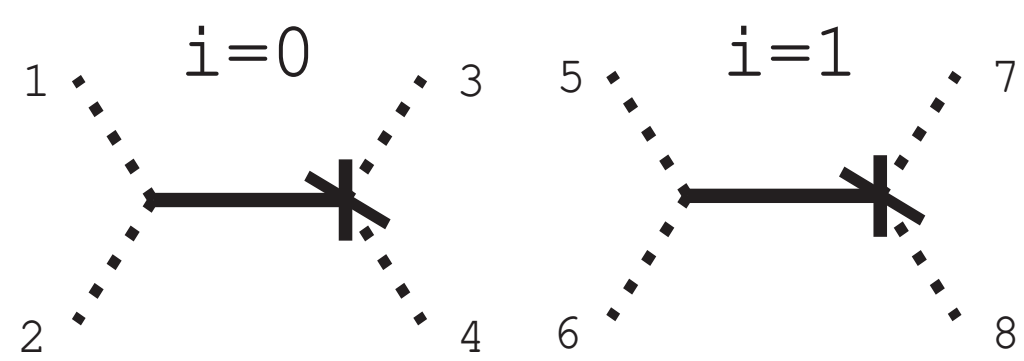

Figure 6: 8-tensor of $\partial_{1} \partial_{2} h_{34} \cdot \partial_{5} \partial_{6} h_{78}$.
$\mathrm{d} 2 \mathrm{~h}[0][1][0]=2$
$\mathrm{d} 2 \mathrm{~h}[1][1][0]=1$
$\mathrm{d} 2 \mathrm{~h}[0][1][1]=2$
$\mathrm{d} 2 \mathrm{~h}[1][1][1]=2$

Generally, contracted suffixes are dummy ones, that is, any suffix-numbers may be taken unless the same number is used for different contractions. In both examples above, the suffixes 1 and 2 are dummy ones and they can be substituted by any non-negative integers, say, 3 and 4 respectively.

\subsection{Code for $(\partial \partial h)^{2}$-Tensors}

The most general $(\partial \partial h)^{2}$-type tensor is the 8-tensor: $\partial_{1} \partial_{2} h_{34} \cdot \partial_{5} \partial_{6} h_{78}$ which is graphically shown in Fig.6. The code for Fig.6 is

$\begin{array}{ll}\mathrm{d} 2 \mathrm{~h}[0][0][0]=1 & \mathrm{~d} 2 \mathrm{~h}[1][0][0]=5 \\ \mathrm{~d} 2 \mathrm{~h}[0][0][1]=2 & \mathrm{~d} 2 \mathrm{~h}[1][0][1]=6 \\ \mathrm{~d} 2 \mathrm{~h}[0][1][0]=3 & \mathrm{~d} 2 \mathrm{~h}[1][1][0]=7 \\ \mathrm{~d} 2 \mathrm{~h}[0][1][1]=4 & \mathrm{~d} 2 \mathrm{~h}[1][1][1]=8\end{array}$

A 4 -tensor $\partial_{1} \partial_{2} h_{34} \cdot \partial_{5} \partial_{6} h_{34}$, which is made from the above 8-tensor(Fig.6) by contracting(connecting) the suffixes (suffix-lines) 3 and 4 with 7 and 8 respectively, is represented as in Fig.7. The code for Fig.7 is
$\mathrm{d} 2 \mathrm{~h}[0][0][0]=1$
$\mathrm{d} 2 \mathrm{~h}[1][0][0]=5$
$\mathrm{d} 2 \mathrm{~h}[0][0][1]=2$
$\mathrm{d} 2 \mathrm{~h}[1][0][1]=6$
$\mathrm{d} 2 \mathrm{~h}[0][1][0]=3$
$\mathrm{d} 2 \mathrm{~h}[1][1][0]=3$
$\mathrm{d} 2 \mathrm{~h}[0][1][1]=4$
$\mathrm{d} 2 \mathrm{~h}[1][1][1]=4$

where two indices 3 and 4 are dummy. There are some other 4-tensors which can be obtained from Fig.6 by connecting two pairs of suffix-lines in different ways.

An invariant: $\partial_{1} \partial_{2} h_{34} \cdot \partial_{1} \partial_{2} h_{34}$, which is made from Fig.7 by contracting suffixes 1 and 2 with 5 and 6 respectively, is graphically obtained as in Fig.8. The code for

\footnotetext{
${ }^{6}$ Because the numbering of two bonds is arbitrary ( permutation symmetry of two $\partial \partial h$-tensors in a $(\partial \partial h)^{2}$-tensor $)$, we may take, say,

$\begin{array}{ll}\mathrm{d} 2 \mathrm{~h}[0][0][0]=5 & \mathrm{~d} 2 \mathrm{~h}[1][0][0]=1 \\ \mathrm{~d} 2 \mathrm{~h}[0][0][1]=6 & \mathrm{~d} 2 \mathrm{~h}[1][0][1]=2 \\ \mathrm{~d} 2 \mathrm{~h}[0][1][0]=7 & \mathrm{~d} 2 \mathrm{~h}[1][1][0]=3 \\ \mathrm{~d} 2 \mathrm{~h}[0][1][1]=8 & \mathrm{~d} 2 \mathrm{~h}[1][1][1]=4\end{array}$
}




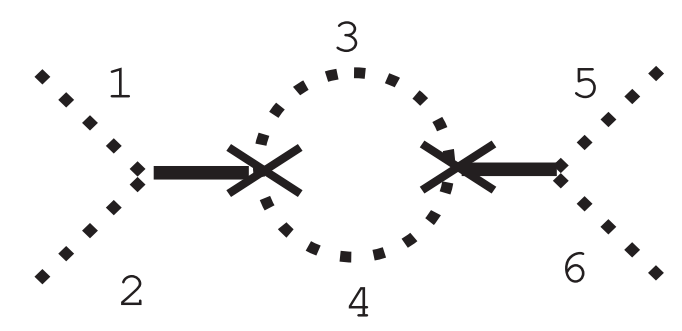

Figure 7: 4-tensor of $\partial_{1} \partial_{2} h_{34} \cdot \partial_{5} \partial_{6} h_{34}$.
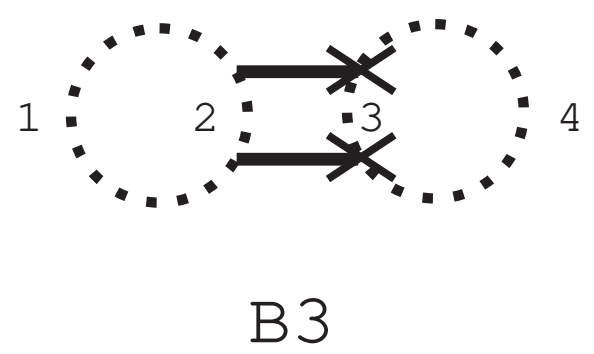

Figure 8: Invariant of $B 3 \equiv \partial_{1} \partial_{2} h_{34} \cdot \partial_{1} \partial_{2} h_{34}$.

Fig. 8 is

\begin{tabular}{|c|c|}
\hline $\mathrm{d} 2 \mathrm{~h}[0][0][0]=1$ & $\mathrm{~d} 2 \mathrm{~h}[1][0][0]=2$ \\
\hline $\mathrm{d} 2 \mathrm{~h}[0][0][1]=2$ & $\mathrm{~d} 2 \mathrm{~h}[1][0][1]=1$ \\
\hline $\mathrm{d} 2 \mathrm{~h}[0][1][0]=4$ & $\mathrm{~d} 2 \mathrm{~h}[1][1][0]=3$ \\
\hline $\mathrm{d} 2 \mathrm{~h}[0][1][1]=3$ & $\mathrm{~d} 2 \mathrm{~h}[1][1][1]=4$ \\
\hline
\end{tabular}

All possible and independent invariants of $(\partial \partial h)^{2}$-type are listed in Appendix A. As mentioned before, all the suffix-lines of the invariants are closed.

\section{Indices for Characterizing $(\partial \partial h)^{2}$-Invariants}

By virtue of the graphical representation, we can identify a tensor by the topology of the corresponding graph. We need not to know the explicit suffix-names associated with the tensor any more. In the computer calculation, this approach reduces the memory space and speeds up the tensor calculation considerably. Let us explain how to do it concretely using the codes explained in the previous section.

The following two items are the important steps for the tensor calculation.

- Listing up all independent invariants (closed graphs).

- Characterizing every closed graph by a set of some appropriate indices, which are computed from the code of the graph. Those indices must be uniquely defined irrespective of arbitrariness of the code due to 1) the suffix permutation symmetry, 2) arbitrariness in numbering bonds, suffix-loops, tadpoles(see later), etc, 3) arbitrariness of dummy suffixes, 4) others (see later). 


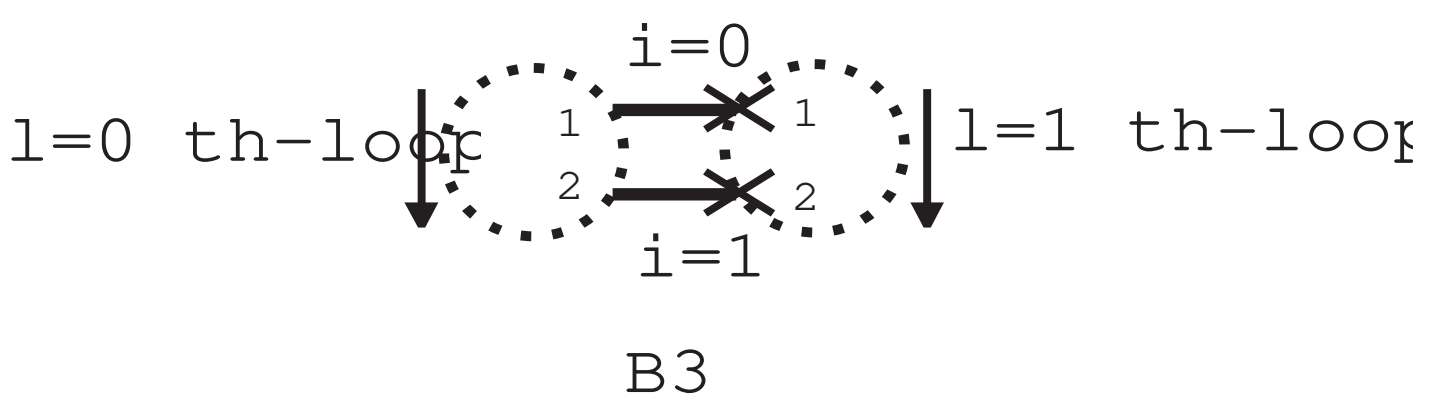

Figure 9: loopstream[ ][][] for B3. Arrows indicate the directions of tracing along each loop.

The former is generally done by the graph theory [15], while the latter can be done by a program using the present algorithm. The indices are explained in this section.

Let us begin with the simplest case of $\partial \partial h$-invariants. See Fig.5. We denote, [the number of suffix-loops] -1, as loop in the program. The index of loop is sufficient for discriminating two independents, $\mathrm{P}$ and $\mathrm{Q}$; loop=1 for $P=\partial_{1} \partial_{1} \overline{h_{22}}$ and loop $=0$ for $Q=\partial_{1} \partial_{2} h_{12}$. In this paper we focus only on $(\partial \partial h)^{2}$-invariants in order to explain the algorithm most simply. The generalization to the general $(\partial \partial h)^{m}$ invariants $(m=1,2,3, \cdots)$ is straightforward. (See Sec.6.) For the $(\partial \partial h)^{2}$-invariants, we can easily check, without the knowledge of the graph theory, that 13 independent invariants listed in App.A exhaust all possible independent ones [14, 16]. Because all suffix-lines for invariants are always closed, we seek some good indices associated with each closed loop. Of course the number of closed loops, loop +1 , is one good index. It is, however, not sufficient to discriminate 13 invariants completely.

\section{1 loop, loopstream [][][] and loopno[ ] [ ]}

A 3-dim array loopstream[maxloopno][2][2], defined below, is initialized as

$$
\begin{gathered}
\text { loopstream }[l][\mathrm{i}][\mathrm{j}]=99, \\
l=0,1, \cdots \quad<\text { maxloopno } \equiv 4 ; \mathrm{i}=0,1 ; \mathrm{j}=0,1
\end{gathered}
$$

For $l$-th loop, we assign an ascending natural number, $1,2,3, \cdots$, at each vertex $(i, j)$ along the loop: loopstream $[l][\mathrm{i}][\mathrm{j}]=1,2,3, \cdots$. B When a vertex $(\mathrm{i}, \mathrm{j})$ belongs to $l$-th loop, we assign as loopno $[\mathrm{i}][\mathrm{j}]=l$. See Fig.9 for example.

loop $=1$

loopstream $[0][0][0]=1 \quad$ loopno $[0][0]=0$

loopstream $[0][0][1]=99 \quad$ loopno[0][1]=1

\footnotetext{
${ }^{7}$ In the following, when the variable names, used in the program, appear in ordinary sentences of the text, they are underlined ('

8 The choice of the starting vertex is arbitrary. This is one of "4) others" in the list of arbitrariness, in the beginning of this section. The number " $i$ " refers to " $i$-th bond" and it runs from 0 to 1 because we are considering a quadratic term of $\partial \partial h$. The number "j" refers to a vertex-type: $\mathrm{j}=0$ means the vertex without the crossing symbol, $\mathrm{j}=1$ means the vertex with it. See Fig.1.
} 


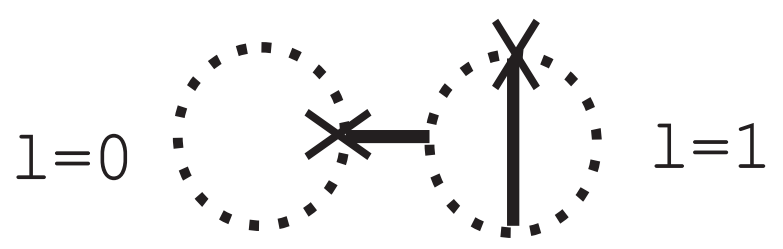

B1

Figure 10: Invariant of $B 1$. \# of vertices of 0 -th and 1-th loop are 1 and 3 respectively. 0 -th loop is a tadpole.

$$
\begin{aligned}
& \text { loopstream }[0][1][0]=2 \quad \text { loopno[1][0] }=0 \\
& \text { loopstream[0][1][1] = 99 loopno[1][1]=1 } \\
& \text { loopstream }[1][0][0]=99 \\
& \text { loopstream[1][0][1] = } 1 \\
& \text { loopstream[1][1][0] }=99 \\
& \text { loopstream[1][1][1] }=2
\end{aligned}
$$

\section{2 maxver []$, \underline{\operatorname{vertex}}[][][]$}

maxver $[l]$ stores the number of vertices which $l$-th loop has. When maxver $\left[l^{\prime}\right]=1$, the $l^{\prime}$-th loop is called tadpole. See Fig.10. maxver[] for Fig.10 is given by

$$
\begin{aligned}
& \operatorname{maxver}[0]=1 \\
& \text { maxver[1] }=3
\end{aligned}
$$

For later convenience, the same information as loopstream[ ][][] is stored in another form: vertex [][][] . When we trace the l-th loop in a certain direction 9 , let the $\mathrm{k}$-th vertex $(\mathrm{k}=0,1, \cdots)$ be $(\mathrm{i}, \mathrm{j})$. Then we assign as vertex $[l][\mathrm{k}][0]=\mathrm{i}$, vertex $[l][\mathrm{k}][1]=\mathrm{j}$, $\mathrm{k}=0,1, \cdots<\operatorname{maxver}[l]$. For Fig.9 we have

$$
\begin{aligned}
\operatorname{vertex}[0][0][0] & =0 \\
\operatorname{vertex}[0][0][1] & =0 \\
\operatorname{vertex}[0][1][0] & =1 \\
\operatorname{vertex}[0][1][1] & =0 \\
\operatorname{vertex}[1][0][0] & =0 \\
\operatorname{vertex}[1][0][1] & =1 \\
\operatorname{vertex}[1][1][0] & =1 \\
\operatorname{vertex}[1][1][1] & =1
\end{aligned}
$$

\section{3 absdeli []$, \underline{\text { absdelj }}[], \underline{\text { tadpoleno }}$ and $\underline{\operatorname{tad}}[\mathrm{]}[\mathrm{]}$}

The number of tadpole-loops in a graph is stored in tadpoleno. When the t-th $(\mathrm{t}=0,1, \cdots<$ tadpoleno $)$ tadpole-loop connects with a bond at a vertex $(\mathrm{i}, \mathrm{j})$, we

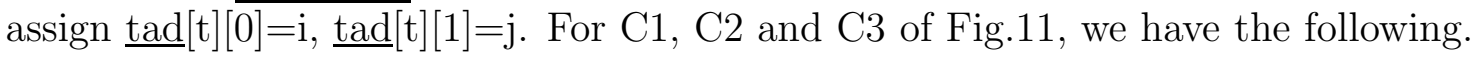

\footnotetext{
${ }^{9}$ The choice of the direction of tracing is another arbitrariness in "4) others" in the beginning of this section.
} 

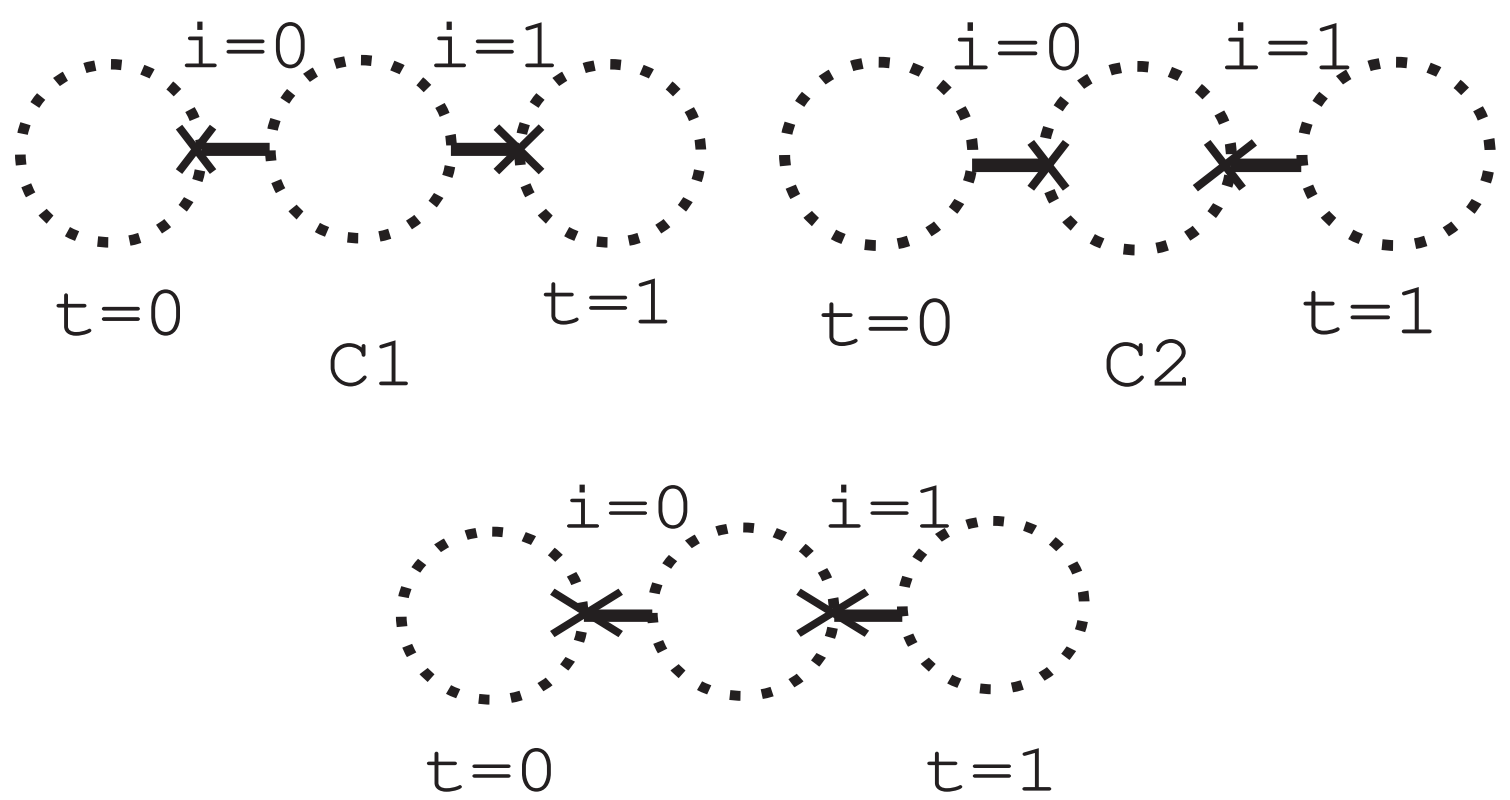

C3

Figure 11: tadpoleno and $\underline{\operatorname{tad}}[][]$ for $C 1, C 2$ and $C 3$.

\section{C1 $\quad$ C2 $\quad$ C3}

tadpoleno $2 \quad 2 \quad 2$

$\operatorname{tad}[0][0] \quad 0 \quad 0 \quad 0$

$\operatorname{tad}[0][1] \quad 1 \quad 001$

$\operatorname{tad}[1][0] \quad 1 \quad 1 \quad 1$

$\operatorname{tad}[1][1] \quad 1 \quad 0 \quad 0$

Now we come to key quantities in this algorithm. When we trace a suffix-line, along a loop, starting from a vertex $\left(\mathrm{i}_{0}, \mathrm{j}_{0}\right)$ in a certain direction, we pass some vertices, $\left(i_{1}, j_{1}\right),\left(i_{2}, j_{2}\right), \cdots$ and finally come back to the starting vertex. We focus on the change of the bond number(i) and the vertex-type number $(\mathrm{j})$ when we pass from a vertex to the next vertex in the above tracing. See Fig.12. For $l$-th loop, we assign as $\sum_{\text {along } l \text {-loop }}|\Delta \mathrm{i}| \equiv \underline{\text { absdeli }}[l], \sum_{\text {along } l \text {-loop }}|\Delta \mathrm{j}| \equiv \underline{\text { absdelj }}[l]$. (They are called bond changing number and vertex changing number respectively, in Ref. [17].) As examples, the indices for A1, A2 and A3 of Fig.13 are given as

\begin{tabular}{|c|c|c|}
\hline & A1 & A2 \\
\hline loop & 0 & 0 \\
\hline$\overline{\text { absdeli }}[0]$ & 4 & 2 \\
\hline absdelj[0] & 2 & 2 \\
\hline
\end{tabular}

absdeli[ ] and absdelj[ ] defined above satisfy the following important properties for programming.

1. They do not depend on the starting vertex for tracing along a loop.

2. They do not depend on the direction of the tracing. 


$$
\text { i=1 }
$$

Figure 12: Change of i (bond number) and $\mathrm{j}$ (vertex-type number). Arrows indicate directions of tracings.

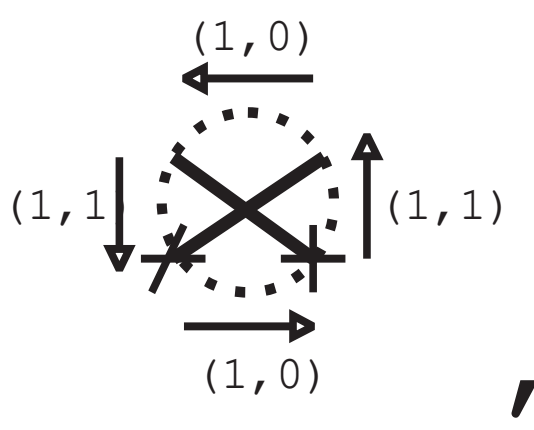

A1

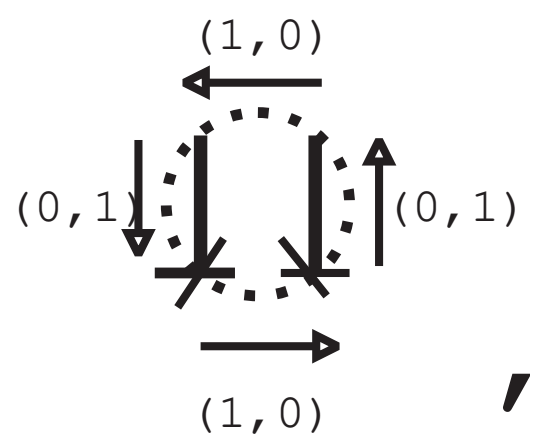

A2

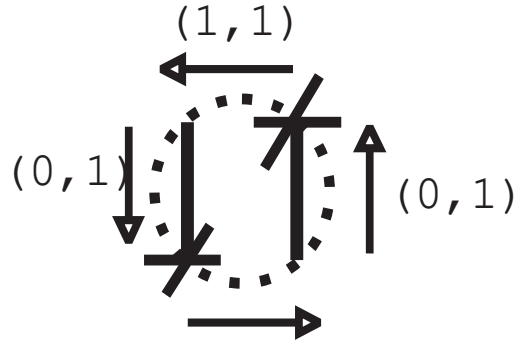

$(1,1)$

A3

Figure 13: Calculation of absdeli[ ] and absdelj[ ] for $A 1, A 2$ and $A 3 .(|\Delta i|,|\Delta j|)$ are listed. 


\subsection{Table of Indices for all invariants}

We give the full list of indices for all invariants in Table 1 . The program, WEAKGRAV, computes all indices for each term, and identify it with a graph following the table. 


\begin{tabular}{|c|c|c|c|c|c|}
\hline Graph,(Run.No) \Indices & loop & tadpoleno & $\underline{\operatorname{tad}[][1]}$ & absdeli & absdelj \\
\hline$A 1=\partial_{\sigma} \partial_{\lambda} h_{\mu \nu} \cdot \partial_{\sigma} \partial_{\nu} h_{\mu \lambda},(0)$ & 0 & 0 & nothing & 4 & 2 \\
\hline$A 2=\partial_{\sigma} \partial_{\lambda} h_{\lambda \mu} \cdot \partial_{\sigma} \partial_{\nu} h_{\mu \nu},(1)$ & 0 & 0 & nothing & 2 & 2 \\
\hline$A 3=\partial_{\sigma} \partial_{\lambda} h_{\lambda \mu} \cdot \partial_{\mu} \partial_{\nu} h_{\nu \sigma},(2)$ & 0 & 0 & nothing & 2 & 4 \\
\hline$B 1=\partial_{\nu} \partial_{\lambda} h_{\sigma \sigma} \cdot \partial_{\lambda} \partial_{\mu} h_{\mu \nu},(3)$ & 1 & 1 & 1 & / & / \\
\hline$B 2=\partial^{2} h_{\lambda \nu} \cdot \partial_{\lambda} \partial_{\mu} h_{\mu \nu},(4)$ & 1 & 1 & 0 & / & / \\
\hline \multirow[b]{2}{*}{$B 3=\partial_{\mu} \partial_{\nu} h_{\lambda \sigma} \cdot \partial_{\mu} \partial_{\nu} h_{\lambda \sigma},(5)$} & \multirow[b]{2}{*}{1} & \multirow[b]{2}{*}{0} & \multirow[b]{2}{*}{ nothing } & 2 & 0 \\
\hline & & & & 2 & 0 \\
\hline \multirow[b]{2}{*}{$B 4=\partial_{\mu} \partial_{\nu} h_{\lambda \sigma} \cdot \partial_{\lambda} \partial_{\sigma} h_{\mu \nu},(6)$} & \multirow[b]{2}{*}{1} & \multirow[b]{2}{*}{0} & \multirow[b]{2}{*}{ nothing } & 2 & 2 \\
\hline & & & & 2 & 2 \\
\hline \multirow[b]{2}{*}{$Q^{2}=\left(\partial_{\mu} \partial_{\nu} h_{\mu \nu}\right)^{2},(7)$} & \multirow[b]{2}{*}{1} & \multirow[b]{2}{*}{0} & \multirow[b]{2}{*}{ nothing } & 0 & 2 \\
\hline & & & & 0 & 2 \\
\hline \multirow{3}{*}{$C 1=\partial_{\mu} \partial_{\nu} h_{\lambda \lambda} \cdot \partial_{\mu} \partial_{\nu} h_{\sigma \sigma},(8)$} & \multirow{3}{*}{2} & \multirow{3}{*}{2} & 1 & $\overline{0}$ & $\overline{0}$ \\
\hline & & & & 2 & 0 \\
\hline & & & 1 & 0 & 0 \\
\hline \multirow{3}{*}{$C 2=\partial^{2} h_{\mu \nu} \cdot \partial^{2} h_{\mu \nu},(9)$} & \multirow{3}{*}{2} & \multirow{3}{*}{2} & 0 & 0 & 0 \\
\hline & & & & 2 & 0 \\
\hline & & & 0 & 0 & 0 \\
\hline \multirow{3}{*}{$C 3=\partial_{\mu} \partial_{\nu} h_{\lambda \lambda} \cdot \partial^{2} h_{\mu \nu},(10)$} & \multirow{3}{*}{2} & \multirow{3}{*}{2} & 1 & 0 & 0 \\
\hline & & & 0 & 0 & 0 \\
\hline & & & & 2 & 2 \\
\hline \multirow{3}{*}{$P Q=\partial^{2} h_{\lambda \lambda} \cdot \partial_{\mu} \partial_{\nu} h_{\mu \nu},(11)$} & \multirow{3}{*}{2} & \multirow{3}{*}{2} & 1 & 0 & 0 \\
\hline & & & 0 & 0 & 0 \\
\hline & & & & 0 & 2 \\
\hline$P^{2}=\left(\partial^{2} h_{\lambda \lambda}\right)^{2},(12)$ & 3 & / & / & / & / \\
\hline
\end{tabular}

Table 1 List of indices for all $(\partial \partial h)^{2}$-invariants. The symbol '/' means 'need not be calculated'. Graph names, $A 1, A 2, \cdots$ are defined in Appendix A. 
Note here that the quantities in Table 1 do not depend on the arbitrariness cited in the beginning of this section. This is one of key points of the present algorithm.

\section{How to use WEAKGRAV}

Let us explain how to use WEAKGRAV taking some simple examples. The program reads all input data from a file (named 'indata.dat'). The input and output data for two examples below are provided in Appendix B.

$\operatorname{Ex} .1 R_{\lambda \mu \nu \sigma} R^{\lambda \mu \nu \sigma}$ as 10

The weak-field expansion $\left(g_{\mu \nu}=\delta_{\mu \nu}+h_{\mu \nu},\left|h_{\mu \nu}\right| \ll 1\right)$ of Riemann tensor is given

$$
\begin{aligned}
2 R_{\lambda \mu \nu \sigma} & =2 R^{\lambda \mu \nu \sigma}+O^{\prime}\left(h^{2}\right) \\
& =\partial_{\mu} \partial_{\nu} h_{\lambda \sigma}-\partial_{\lambda} \partial_{\nu} h_{\mu \sigma}-\nu \leftrightarrow \sigma+O\left(h^{2}\right) .
\end{aligned}
$$

Changing the Greek suffixes $(\mu, \nu, \lambda, \sigma)$ to non-negative integers, say, $(1,2,3,4)$, we can get the input data of (termnoa, weightd2ha[ ],d2ha[ ][ ][ ]) for $R_{\lambda \mu \nu \sigma}$ and (termnob, weightd2hb[ ],d2hb[][][ ]) for $R^{\lambda \mu \nu \sigma}$.

The output says

$$
2 R_{\lambda \mu \nu \sigma} \times 2 R^{\lambda \mu \nu \sigma}=-8 \times \mathrm{A} 1+4 \times \mathrm{B} 3+4 \times \mathrm{B} 4+O\left(h^{2}\right),
$$

where $A 1=\partial_{\sigma} \partial_{\lambda} h_{\mu \nu} \cdot \partial_{\sigma} \partial_{\nu} h_{\mu \lambda}, B 3=\partial_{\mu} \partial_{\nu} h_{\lambda \sigma} \cdot \partial_{\mu} \partial_{\nu} h_{\lambda \sigma}, B 4=\partial_{\mu} \partial_{\nu} h_{\lambda \sigma} \cdot \partial_{\lambda} \partial_{\sigma} h_{\mu \nu}$ (Table 1,Appendix A).

$\operatorname{Ex} .2 R_{\mu \nu} R^{\mu \nu}$

$R_{\mu \nu}$ and $R^{\mu \nu}$ is expanded as

$$
\begin{aligned}
2 R_{\mu \nu} & =2 R^{\mu \nu}+O^{\prime}\left(h^{2}\right) \\
& =\partial_{\mu} \partial_{\nu} h_{\alpha \alpha}-\partial_{\mu} \partial_{\alpha} h_{\alpha \nu}-\partial_{\nu} \partial_{\alpha} h_{\alpha \mu}+\partial^{2} h_{\mu \nu}+O\left(h^{2}\right) .
\end{aligned}
$$

Changing the Greek suffixes $(\mu, \nu, \alpha)$ to $(1,2,3)$ for termnoa, weightd2ha[ ] and d2ha[ ][ ][ ] (input data for $\left.2 R_{\mu \nu}\right)$ and changing $(\mu, \nu, \alpha)$ to $(1,2,4)$ for termnob, weightd2hb[ ] and $\mathrm{d} 2 \mathrm{hb}\left[\mathrm{]}\left[\mathrm{]}\left[\mathrm{]}\right.\right.\right.$ (input data for $2 R^{\mu \nu}$ ), we obtain the the input data.

The output says

$$
\begin{aligned}
2 R_{\mu \nu} \times 2 R^{\mu \nu}= & 2 \times \mathrm{A} 2+2 \times \mathrm{A} 3-4 \times \mathrm{B} 1-4 \times \mathrm{B} 2 \\
+1 & \times \mathrm{C} 1+1 \times \mathrm{C} 2+2 \times \mathrm{C} 3+O\left(h^{2}\right)
\end{aligned}
$$

where $A 2=\partial_{\sigma} \partial_{\lambda} h_{\lambda \mu} \cdot \partial_{\sigma} \partial_{\nu} h_{\mu \nu}, A 3=\partial_{\sigma} \partial_{\lambda} h_{\lambda \mu} \cdot \partial_{\mu} \partial_{\nu} h_{\nu \sigma}, B 1=\partial_{\nu} \partial_{\lambda} h_{\sigma \sigma} \cdot \partial_{\lambda} \partial_{\mu} h_{\mu \nu}, B 2=$ $\partial^{2} h_{\lambda \nu} \cdot \partial_{\lambda} \partial_{\mu} h_{\mu \nu}, C 1=\partial_{\mu} \partial_{\nu} h_{\lambda \lambda} \cdot \partial_{\mu} \partial_{\nu} h_{\sigma \sigma}, C 2=\partial^{2} h_{\mu \nu} \cdot \partial^{2} h_{\mu \nu}, C 3=\partial_{\mu} \partial_{\nu} h_{\lambda \lambda} \cdot \partial^{2} h_{\mu \nu}$, (Table 1,Appendix A).

\footnotetext{
${ }^{10}$ See [14] for the present convention of gravitational quantities.
} 


\section{Discussion and Conclusion}

When we do the suffix contraction in usual algebraic softwares, such as REDUCE, we cannot help expressing the suffix permutation symmetry by explicitly writing up all possible permuted cases. For example, in REDUCE, Q defined in Sec.2 is coded as

INTEGER M,N;

REAL Q;

FOR ALL M,N LET $\quad$ DDH $[\mathrm{M}, \mathrm{N}, \mathrm{M}, \mathrm{N}]=\mathrm{Q}$;

FOR ALL M,N LET $\quad \mathrm{DDH}[\mathrm{M}, \mathrm{N}, \mathrm{N}, \mathrm{M}]=\mathrm{Q}$;

where an array DDH[M,N,L,S] expresses $\partial_{\mu} \partial_{\nu} h_{\lambda \sigma}$. It is very hard to generalize this approach to higher-power terms or to higher-rank tensors because it directly deals with all suffixes. The number of programming lines for the definition of each invariant increases rapidly. The present proposed approach do not treat suffixes directly, but the topology of graphs (, in other words, connectivity of suffix lines). The good efficiency of the algorithm based on the idea has been checked by some concrete programs.

In this paper we have explained the new algorithm using a simple invariants of $(\partial \partial h)^{2}$. Its generalization to other types can be done in a similar way. As the order of invariants increases, additional indices are required to discriminate between invariants (graphs). In [17], we have used a generalized version of this program and have obtained the weak-field expansion of different products of three Riemann tensors. We have treated $(\partial \partial h)^{3}$-invariants (totally 90 terms) and the four indices are additionally required: 1) Vertex-Type Order, 2) Number of $\partial \partial$-vertices and $h$ vertices, 3) Number of crossing, 4) Disconnectivity. Most general SO(n) tensors (including invariants) constructed from $\partial h, \partial \partial h, \partial \partial \partial h, \cdots$ can be treated similarly. The point in this algorithm is to find good indices to discriminate between invariants.

The present algorithm is a new efficient way to do the suffix contraction using a computer. It can be applied not only to gravitational theories but also to general field theories as stated in Sec.1. One of interesting applications to physics is the Weyl anomaly calculation in gravitational theories. It is known that $(\partial \partial h)^{m}$ - type tensor calculation is sufficient for the Weyl anomaly calculation in 2m-dim gravitational theories. It was confirmed that Weyl anomaly in 4 dim quantum gravity is obtained by the present program 14.

\section{Acknowledgement}

The author thanks Dr. N. Ikeda (RIMS, , Kyoto Univ.) for discussions on Weyl anomaly problem in gravitational theories. He also thanks Prof. J.A.M. Vermaseren (NIKHEF-H,Amsterdam) for reading the manuscript and for some comments. 

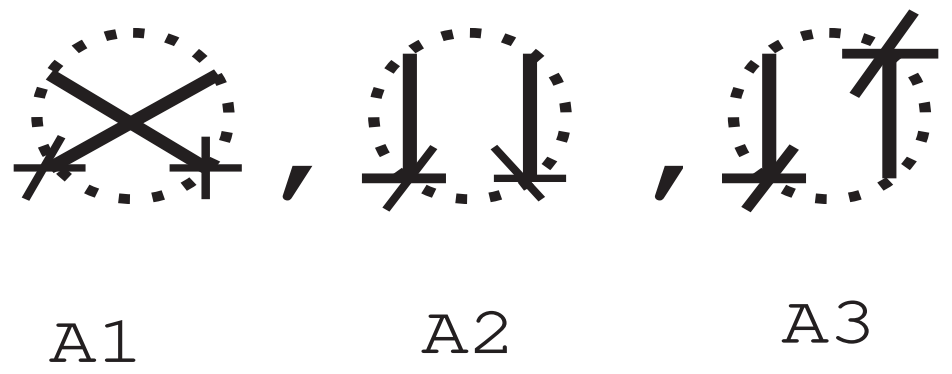

Figure 14: Graphs of $(\partial \partial h)^{2}$-invariants, loop no $=1$.

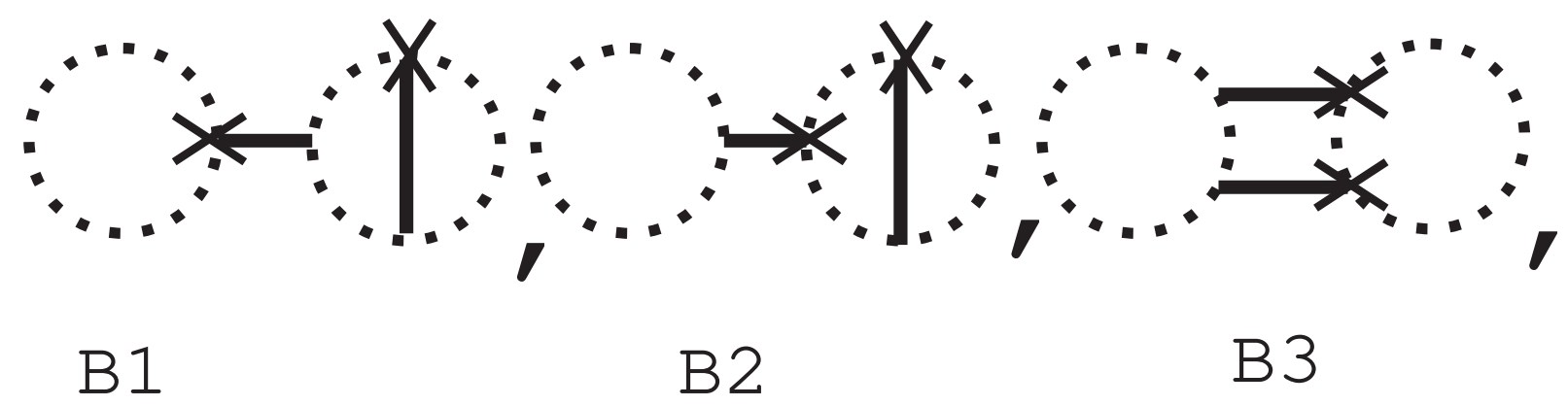

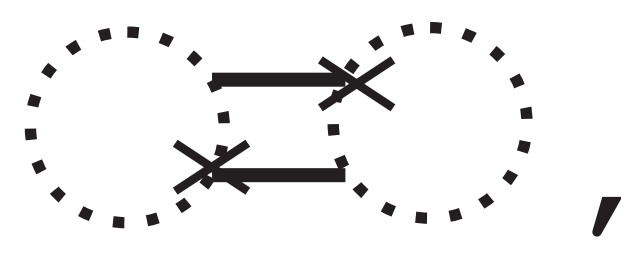

B 4

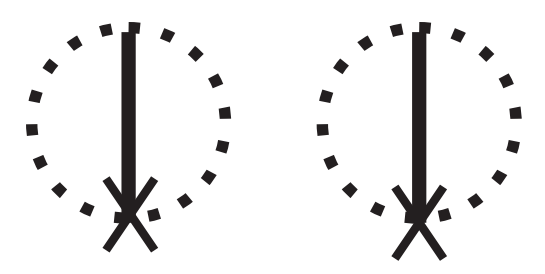

QQ

Figure 15: Graphs of $(\partial \partial h)^{2}$-invariants, loop no $=2$.

\section{Appendix A. Graphical Representation of $(\partial \partial h)^{2}$-Invariants}

In the weak field expansion of n-dim Euclidean gravity : $g_{\mu \nu}=\delta_{\mu \nu}+h_{\mu \nu},|h| \ll 1$, we must generally treat global SO(n)-tensors which are composed of $h_{\mu \nu}, \partial_{\alpha} h_{\mu \nu}$, $\partial_{\alpha} \partial_{\beta} h_{\mu \nu}, \cdots$. Let us focus here on those tensors which are composed only of $\partial_{\alpha} \partial_{\beta} h_{\mu \nu}$. We introduce its graphical representation as shown in Fig.1 in Sec.2 of the text. Contraction of suffixes is graphically represented by connecting the dotted lines with the same suffix. For example see Fig.2 and 3 of Sec.2

In Sec.2 we have graphically presented $\partial h$-invariants (Fig.3). They are $\mathrm{P}=\partial^{2} h$, $\mathrm{Q}=\partial_{\mu} \partial_{\nu} h_{\mu \nu}$. Similarly we can list up all independent $(\partial \partial h)^{2}$-invariants as follows. They are grouped by the number of the suffix-loop: loop no $=1$, Fig. 14 ; loop no $=2$ : Fig.15; loop no =3: Fig.16; loop no =4: Fig.17;. 


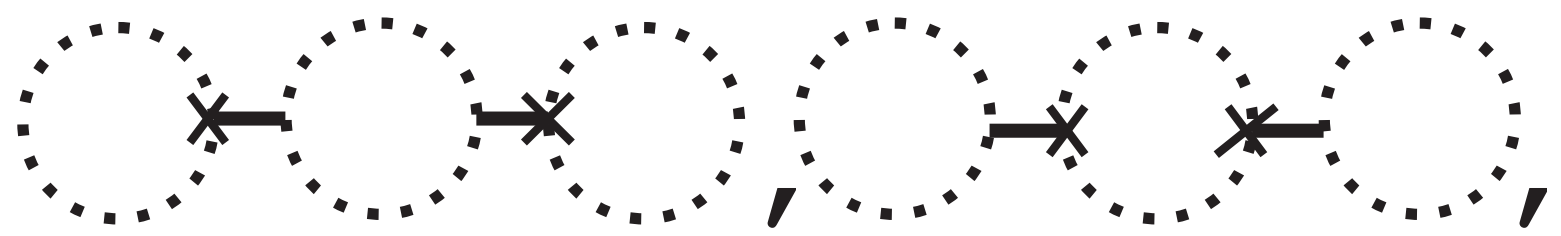

C1

C2

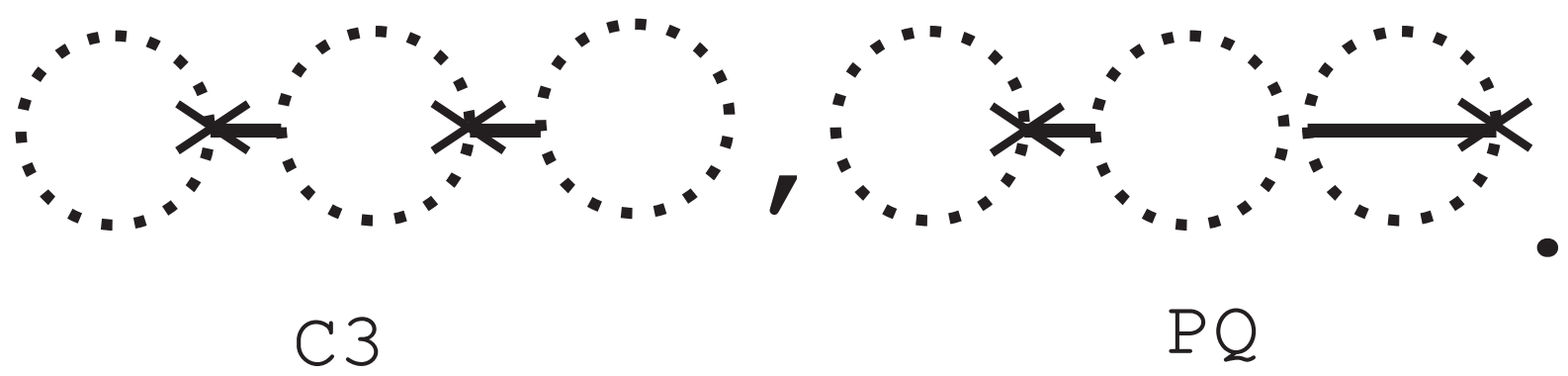

Figure 16: Graphs of $(\partial \partial h)^{2}$-invariants, loop no=3.

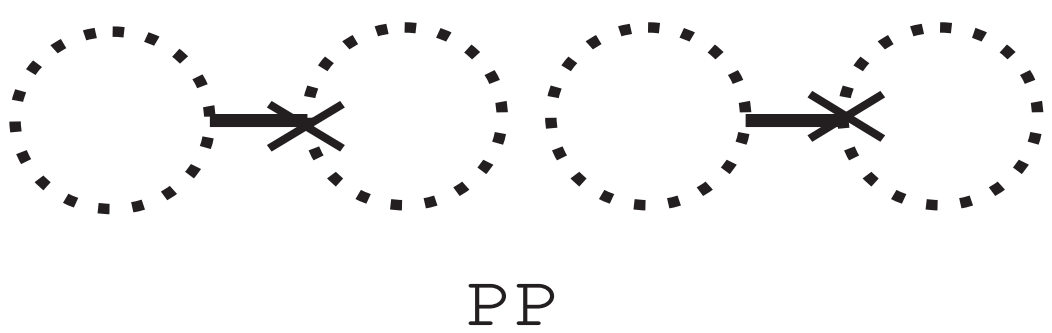

Figure 17: Graphs of $(\partial \partial h)^{2}$-invariants, loop no $=4$. 
We have totally 13 invariants (10 connected, 3 disconnected). Their literal expressions are listed in Table 1 of the text(Subsec.4.4). 


\section{Appendix B. Input and Output Data of Sample Calculations}

Example 1, Input Data

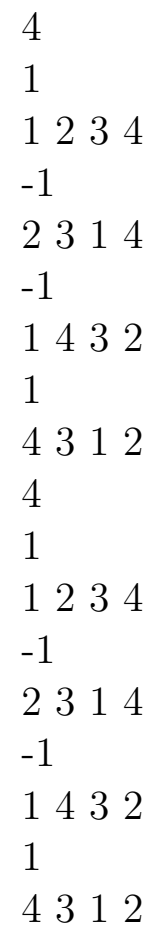

4

1

1234

$-1$

2314

$-1$

1432

1

4312

4

1

1234

$-1$

2314

$-1$

1432

1

4312

Example 1, Output Data

start of MAIN

Input Check

termnoa $=4$

term 0 , weight $=1$

d2ha[0][0][0]=1

d2ha[0][0][1]=2

d2ha $[0][1][0]=3$

d $2 \mathrm{ha}[0][1][1]=4$

term 1 ,weight $=-1$

d2ha[1][0][0]=2

d2ha[1][0][1]=3

d2ha[1][1][0]=1

d2ha[1][1][1] =4

term 2 , weight $=-1$

d2ha[2][0][0]=1

d2ha[2][0][1] =4

d2ha[2][1][0]=3 


$$
\begin{aligned}
& \mathrm{d} 2 \mathrm{ha}[2][1][1]=2 \\
& \text { term3, weight }=1 \\
& \text { d2ha [3][0][0] =4 } \\
& \text { d2ha [3][0][1]=3 } \\
& \text { d2ha[3][1][0]=1 } \\
& \text { d2ha[3][1][1] =2 } \\
& \text { termnob }=4 \\
& \text { term0, weight }=1 \\
& \mathrm{~d} 2 \mathrm{hb}[0][0][0]=1 \\
& \mathrm{~d} 2 \mathrm{hb}[0][0][1]=2 \\
& \mathrm{~d} 2 \mathrm{hb}[0][1][0]=3 \\
& \mathrm{~d} 2 \mathrm{hb}[0][1][1]=4 \\
& \text { term } 1 \text {, weight }=-1 \\
& \mathrm{~d} 2 \mathrm{hb}[1][0][0]=2 \\
& \mathrm{~d} 2 \mathrm{hb}[1][0][1]=3 \\
& \mathrm{~d} 2 \mathrm{hb}[1][1][0]=1 \\
& \mathrm{~d} 2 \mathrm{hb}[1][1][1]=4 \\
& \text { term2, weight }=-1 \\
& \mathrm{~d} 2 \mathrm{hb}[2][0][0]=1 \\
& \mathrm{~d} 2 \mathrm{hb}[2][0][1]=4 \\
& \mathrm{~d} 2 \mathrm{hb}[2][1][0]=3 \\
& \mathrm{~d} 2 \mathrm{hb}[2][1][1]=2 \\
& \text { term } 3 \text {, weight }=1 \\
& \mathrm{~d} 2 \mathrm{hb}[3][0][0]=4 \\
& \mathrm{~d} 2 \mathrm{hb}[3][0][1]=3 \\
& \mathrm{~d} 2 \mathrm{hb}[3][1][0]=1 \\
& \mathrm{~d} 2 \mathrm{hb}[3][1][1]=2
\end{aligned}
$$

\section{FINAL ANSWER}

$-8 \mathrm{~A} 1$

0 A2

0 A3

0 B1

0 B2

4 B3

4 B4

0 QQ

$0 \mathrm{C} 1$

$0 \mathrm{C} 2$

$0 \mathrm{C} 3$

0 PQ

$0 \mathrm{PP}$ 
Example 2, Input Data

4

1

1233

$-1$

1332

$-1$

2331

1

3312

4

1

1244

$-1$

1442

$-1$

2441

1

4412

Example 2, Output Data

start of MAIN

Input Check

termnoa $=4$

term 0 , weight $=1$

$\mathrm{d} 2 \mathrm{ha}[0][0][0]=1$

d2ha [0][0][1] =2

$\mathrm{d} 2 \mathrm{ha}[0][1][0]=3$

d 2 ha $[0][1][1]=3$

term 1 , weight $=-1$

d2ha[1][0][0]=1

$\mathrm{d} 2 \mathrm{ha}[1][0][1]=3$

d2ha[1][1][0]=3

d2ha[1][1][1] =2

term2, weight $=-1$

$\mathrm{d} 2 \mathrm{ha}[2][0][0]=2$

d 2 ha $[2][0][1]=3$

$\mathrm{d} 2 \mathrm{ha}[2][1][0]=3$

d2ha[2][1][1] =1

term3, weight $=1$ 


$$
\begin{aligned}
& \text { d2ha[3][0][0]=3 } \\
& \text { d2ha[3][0][1]=3 } \\
& \text { d2ha[3][1][0]=1 } \\
& \text { d2ha[3][1][1]= }=2 \\
& \text { termnob=4 } \\
& \text { term0,weight=1 } \\
& \text { d2hb[0][0][0]=1 } \\
& \text { d2hb[0][0][1]=2 } \\
& \text { d2hb[0][1][0]=4 } \\
& \text { d2hb[0][1][1]=4 } \\
& \text { term1,weight=-1 } \\
& \text { d2hb[1][0][0]=1 } \\
& \text { d2hb[1][0][1]=4 } \\
& \text { d2hb[1][1][0]=4 } \\
& \text { d2hb[1][1][1]=2 } \\
& \text { term2,weight=-1 } \\
& \text { d2hb[2][0][0]=2 } \\
& \text { d2hb[2][0][1]=4 } \\
& \text { d2hb[2][1][0]=4 } \\
& \text { d2hb[2][1][1]=1 } \\
& \text { term3,weight=1 } \\
& \text { d2hb[3][0][0]=4 } \\
& \text { d2hb[3][0][1]=4 } \\
& \text { d2hb[3][1][0]=1 } \\
& \text { d2hb[3][1][1]=2 }
\end{aligned}
$$

\section{FINAL ANSWER}

0 A1

2 A2

$2 \mathrm{~A} 3$

-4 B1

$-4 \mathrm{~B} 2$

0 B3

0 B4

0 QQ

$1 \mathrm{C} 1$

$1 \mathrm{C} 2$

$2 \mathrm{C} 3$

$0 \mathrm{PQ}$

$0 \mathrm{PP}$ 


\section{References}

[1] R.P.Feynman,Acta Phys.Polonica24(1963)697

[2] G.'tHooft and M.Veltman,Ann.Inst.Henri Poincaré20(1974)69

[3] M.H.Goroff and A.Sagnotti,Phys.Lett.150B(1985)81; Nucl.Phys.B266(1986)709

[4] A.E.M. van de Ven,Nucl.Phys.B378(1992)309

[5] J.A.M.Vermaseren,Symbolic Manipulation with FORM, Tutorial and Reference Manual ver 2,1991 (Amsterdam: Computer Algebra Nederland); Version 1.0 of the program is available via anonymous ftp from nikhef.nikhef.nl

[6] S.Ichinose,Phys.Lett.B284(1992)234;Nucl.Phys.B395(1993)433

[7] W.A.Bardeen and B.Zumino,Nucl.Phys.B244(1984)421

[8] L.Bonora,P.Pasti and M.Bregola,Class.Quantum Grav.3(1986)635.

[9] S.Deser and A.Schwimmer,Phys.Lett.B309(1993)279.

[10] T.Arakelyan,D.R.Karakhanyan,R.P.Manvelyan and R.L.Mkrtchyan, Phys.Lett.B353(1995)52.

[11] D.R.Karakhanyan,R.P.Manvelyan and R.L.Mkrtchyan,Mod.Phys.Lett.A11(1996)409.

[12] N.D.Birrel and P.C.Davies,Quantum Fields in Curved Space, 1982,Cambridge Univ.Press.

[13] S.Ichinose,Class.Quantum Grav.12,1021(1995).

[14] S.Ichinose and N.Ikeda, Phys.Rev.D53,5932(1996).

[15] F.Harary,Graph Theory, Reading-Menlo Park-Ontario, Addison-Wesley Pub.Co., 1969.

[16] S.Ichinose and N.Ikeda, Preprint of Univ.of Shizuoka,US-96-03, hep-th/9609013 , 'Weak Field Expansion of Gravity and Graphical Representation'

[17] S.Ichinose and N.Ikeda, Jour.Math.Phys.38,6475(1997), hep-th/9702003 


\section{Figure Captions}

- Fig.1 4-tensor $\partial_{\mu} \partial_{\nu} h_{\alpha \beta}$

- Fig.2 2-tensors of (a) $\partial^{2} h_{\alpha \beta}$, (b) $\partial_{\mu} \partial_{\nu} h_{\alpha \alpha}$ and (c) $\partial_{\mu} \partial_{\beta} h_{\alpha \beta}$

- Fig.3 Invariants of $P \equiv \partial_{\mu} \partial_{\mu} h_{\alpha \alpha}$ and $Q \equiv \partial_{\alpha} \partial_{\beta} h_{\alpha \beta}$.

- Fig.4 4-tensor of $\partial_{1} \partial_{2} h_{34}$.

- Fig.5 Invariants of $P=\partial_{1} \partial_{1} h_{22}$ and $Q=\partial_{1} \partial_{2} h_{12}$.

- Fig.6 8-tensor of $\partial_{1} \partial_{2} h_{34} \cdot \partial_{5} \partial_{6} h_{78}$.

- Fig.7 4-tensor of $\partial_{1} \partial_{2} h_{34} \cdot \partial_{5} \partial_{6} h_{34}$.

- Fig.8 Invariant of $B 3 \equiv \partial_{1} \partial_{2} h_{34} \cdot \partial_{1} \partial_{2} h_{34}$.

- Fig.9 loopstream[ ][ ][ ] for B3.

- Fig.10 Invariant of B1. \# of vertices of 0-th and 1-th loop are 1 and 3 respectively. 0 -th loop is a tadpole.

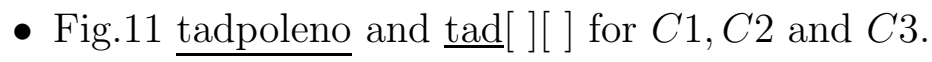

- Fig. 12 Change of i (bond number) and j (vertex-type number). Arrows indicate directions of tracings.

- Fig.13 absdeli[ ] and absdelj[] for $A 1, A 2$ and $A 3$.

- Fig.14 Graphs of $(\partial \partial h)^{2}$-invariants, loop no=1.

- Fig.15 Graphs of $(\partial \partial h)^{2}$-invariants, loop no=2.

- Fig.16 Graphs of $(\partial \partial h)^{2}$-invariants, loop no=3.

- Fig.17 Graphs of $(\partial \partial h)^{2}$-invariants, loop no=4. 